\title{
Von Dashboards zu Dashboarding
}

Bei der Entwicklung von Dashboards stehen Visualisierungsaspekte oft im Vordergrund. Dabei sind Prozessaspekte für erfolgreiches Dashboard Design mindestens ebenso wichtig. Eine WHU-Studie bei Software-Anbieter SAP identifiziert wesentliche Faktoren für effektiv nutzbare Dashboards und schafft damit die Basis für ein erweitertes, prozessorientiertes Verständnis von Dashboards.

Marc Feldmann, Robert Weller 
In einer Zeit, in der wachsende Datenmengen die Anpassungsfähigkeit des Controllings auf die Probe stellen (vergleiche Schäffer/Weber 2016, S. 9 f.) und ständig neue, teils konkurrierende Perspektiven auf das organisationale Geschehen eröffnen, wünschen sich viele Entscheiderinnen und Entscheider vor allem eines: Klarheit. Sie möchten entscheidungsrelevante Muster in den Strömen betriebswirtschaftlicher Informationen schnell und einfach identifizieren. Genau diesen Wunsch adressieren Dashboards (vergleiche Weber/Schäffer 2020, S. 106 ff.). Ihre Anziehungskraft für die Unternehmensführung spiegelt sich in ihrer Verbreitung im deutschsprachigen Raum wider. Eine Studie des WHU Controller Panels zeigt, dass bereits 201939 Prozent der befragten Organisationen Dashboards nutzten, weitere 31 Prozent planten die Nutzung (vergleiche Reimer/Schäffer/Weber 2019). Das Wachstum des Marktes für Lösungen zur Datenanalyse um 10,4 Prozent im selben Jahr bestätigte diesen Trend auf globaler Ebene (vergleiche Gartner 2020). Selbst in der Kommunikation krisenrelevanter Informationen an die Öffentlichkeit vertrauen Experten zunehmend auf Dashboards (vergleiche Johns Hopkins University 2020). Die Implementierung von Dashboards ist jedoch nicht gleichzusetzen mit deren erfolgreicher Nutzung.

Die Basis dafür, wie und ob Dashboards genutzt werden, legt bereits ihre Entwicklung. Unter anderem so griffige Phrasen wie „Above all else show the data" des Visualisierungspioniers Edward Tufte (2001, S. 92) tragen jedoch dazu bei, dass in der Entwicklungsphase die Aufmerksamkeit oft vor allem visuellen Design-Aspekten gilt, während wichtige, häufig aber weniger greifbare Prozessaspekte aus dem Blickfeld geraten. Eine beim Software-Hersteller SAP 2019 und 2020 durchgeführte Dashboard-Studie macht dies deutlich. Insbesondere der Blick auf die Nutzung eines Dashboards zur unternehmensweiten Mitarbeiterführung und -entwicklung machte hier deutlich, dass eine Fokussierung auf Design-Aspekte allein nicht ausreicht, um eine Dashboard-Lösung erfolgreich zu implementieren. Die Nutzer lobten zwar die grafische Oberfläche des Dashboards und nahmen es als merkliche Verbesserung gegenüber früheren Steuerungsinstrumenten wahr. In der Befragung zeigte sich jedoch, dass auch andere Aspekte Berücksichtigung finden müssen, damit alle intendierten Nutzer es auch regelmäßig verwenden.

Das visuelle Design ist demnach wichtig, doch muss der Fokus von Entwicklungsbeginn an erweitert werden. Auf Grundlage der Studienergebnisse haben wir deshalb vier Empfehlungen für eine erfolgreiche Konzeption und Implementierung von Dashboards erarbeitet.

\section{Klare Vermittlung von Zweck und Relevanz}

Zunächst legte unsere Studie offen, dass manche der untersuchten Dashboards merklich weniger genutzt wurden als andere. Nach den Gründen gefragt, erklärten die Nutzer Unterschiede zum einen damit, dass ihnen die mit dem in der Studie fokussierten Dashboard verbundene Strategie nicht vollständig klar war. Zum anderen konnten sie trotz einer frühen Einbindung in die Entwicklung des Dashboards nicht genau benennen, welche operativen Bedarfe das Dashboard beantworten sollte. Für die intendierten Nut-

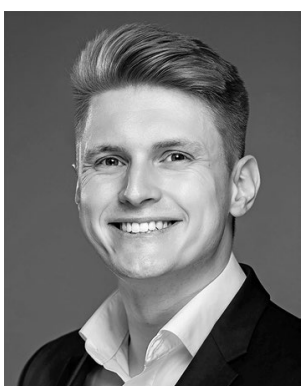

\section{Marc Feldmann}

ist Doktorand am Institut für Management und Controlling (IMC) der WHU Otto Beisheim School of Management. E-Mail:marc.feldmann@whu.edu

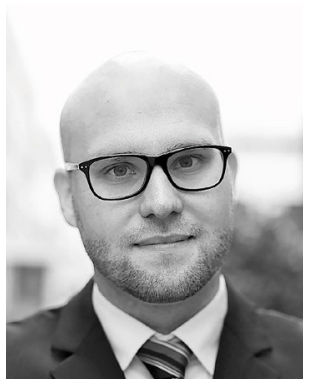

Robert Weller ist Chief Operating Officer im Bereich Intelligent Data \& Analytics bei SAP SE sowie Evangelist für Analytics und Agile Leadership.

E-Mail: robert.weller@sap.com 
Der ausgeprägte Fokus auf Visualisierung droht, wichtige Prozessaspekte von Dashboards zu marginalisieren. zer war nicht wirklich erkennbar, welche Relevanz das Dashboard für ihre konkrete Arbeit hatte. Das bedeutete, dass sie nicht wussten, was sie mithilfe des Dashboards anders machen sollten als bisher, das heißt, wie sie ihr Verhalten zu ändern hatten. Es war für sie mitunter nicht nachvollziehbar, wie sie mit Entscheidungen in ihrem Geschäftsalltag die im Dashboard gezeigten Kennzahlen konkret beeinflussen könnten. So pointierte etwa ein Bereichsleiter: „Die Dinge, die mein Chef mir auf den Tisch legt, die Diskussionen, die ich betriebswirtschaftlich habe, passen nicht zu den Dashboards und zu den Inhalten.“

\section{Empfehlung 1}

Diese Beobachtungen verdeutlichen: Um Nutzern den Zweck eines Dashboards zu vermitteln, muss das Projekt-Team die mit dem Roll-out verbundene strategische Zielsetzung sowie beabsichtigte Verhaltensänderungen von Beginn an klar und transparent kommunizieren. Dazu müssen einerseits die Nutzer ganz konkret wissen, was von ihnen erwartet wird. Andererseits müssen das Projekt-Team und reichweitenstarke Sponsoren aus dem oberen Management, also diejenigen, die das Projekt unternehmensintern treiben, präzise und vollumfänglich deutlich machen, weshalb sie das Dashboard einführen. Auch müssen sie deutlich machen, weshalb die dort dargestellten Kennzahlen relevant sind (vergleiche Sabherwal/Jeyaraj/Chowa 2006, S. 1862). Den Nutzern muss klar werden, dass die Kennzahlen für ihren Bereich verbindlich und aussagekräftig sind und dass sie diese beeinflussen können.

\section{„Das beste Dashboard ist eines, das zum Nutzer kommt - und zwar immer und nur dann, wenn Handlungsbedarf besteht."}

Um dies sicherzustellen und die Relevanz von Dashboards für konkrete Aufgabenkontexte zu demonstrieren, eignen sich reale Fallbeispiele. Sie helfen den intendierten Nutzern zu verstehen, wie sie mithilfe des Dashboards ihren Berichtspflichten besser nachkommen und Probleme im Geschäftsalltag lösen können. Dabei sollten die Nutzer von Führungskräften schrittweise und medial unterstützt durch diese Fallbeispiele geführt werden. Besonders als Multiplikatoren geeignet sind die Führungskräfte, die in der Organisation sichtbar sind und häufig und persönlich mit Mitgliedern der Nutzergruppe interagieren.

Das Projekt-Team kann die Relevanz für die Nutzer durch weitere Maßnahmen zusätzlich erhöhen. So können beispielsweise die Ist-Zahlen im Dashboard um aussagekräftige Benchmarks, Trends und Zielwerte ergänzt werden. Außerdem sollte klar sein, welche Dashboard-Inhalte zur interaktiven Nutzung bestimmt sind, also ständig vom Management im Blick behalten werden müssen, und welche zur diagnostischen Nutzung, also keiner fortwährenden Aufmerksamkeit bedürfen (vergleiche Simons 1995). Gera- 
de bei Letzterer kann es hilfreich sein, wenn das Dashboard Nutzer automatisiert über konkrete Handlungsbedarfe informiert (vergleiche Yigitbasioglu/Velcu 2012, S. 47 f.). Dies kann in Form von E-Mails oder Pop-upBenachrichtigungen geschehen, die bei Schwellwertüberschreitungen an Nutzer gesendet werden und idealerweise per direktem Zugriffslink zum entsprechenden Dashboard-Inhalt führen. Diese Schwellwerte können zum einen durch Nutzer frei definierbar sein, zum anderen können parallel durch Analyse historischer Muster adäquate Schwellwerte vom Dashboard vorgeschlagen werden. Hier gilt: Das beste Dashboard ist eines, das zum Nutzer kommt - und zwar immer und nur dann, wenn Handlungsbedarf besteht.

\section{Unterscheidung von Kern und Peripherie}

In unserer Fallstudie gehörten neben Führungskräften auch Mitarbeiter in geschäftsnahen, unterstützenden Human-Resources-Funktionen (HR) zur Nutzergruppe des Dashboards. Beide Teilgruppen hatten ihre eigenen spezifischen Anforderungen an das Dashboard.

Das Dilemma: Um die Akzeptanz zu erhöhen und die beabsichtigte Verhaltensbeeinflussung zu maximieren, sollte ein Dashboard möglichst viele Nutzer möglichst gezielt ansprechen. Je mehr Nutzer aber angesprochen werden, desto heterogener werden tendenziell auch deren Anforderungen, sodass das Dashboard weniger gezielt in der Lage ist, diese zu bedienen. Das wiederum kann die Nutzerzufriedenheit verringern und dazu führen, dass das Dashboard weniger verwendet wird. Der Kommentar eines Nutzers illustriert die Problematik: „Dem Gelegenheitsnutzer wird im Dashboard sehr viel zur Verfügung gestellt, von dem er die Finger lässt aus Angst, etwas kaputt zu machen. Und jemand, der die komplette Funktionalität nutzen will, bräuchte ein zweistündiges Einführungsvideo." Das untersuchte Dashboard enthielt etwa Kennzahlen zu regionalen Unterschieden zwischen Gehaltsniveaus. Für Nutzer, die weder regionsübergreifende Teams führen, noch Budgetverantwortung haben, noch zu Gehaltsverhandlungen befugt sind, ist dieser Teil des Dashboards aber nicht wirklich relevant.

\section{Empfehlung 2}

Je heterogener eine Nutzergruppe ist, desto schwieriger kann es sein, Akzeptanz für das Dashboard zu erhalten. Die Heterogenität kann sich dabei in unterschiedlichen funktionalen Rollen, Verantwortlichkeiten, Nutzungspräferenzen und Möglichkeiten zur Einflussnahme auf die in Dashboards dargestellten Kennzahlen manifestieren. Sie führt dazu, dass die Harmonisierung verschiedener Nutzeranforderungen erschwert wird, insbesondere wenn die Dashboard-Inhalte statisch sind und so nicht dynamisch reagieren können. Eine mögliche Lösung für dieses Problem kann eine nutzersegmentbasierte Modularisierung des Dashboards sein, die in zwei Schritten abläuft.

Im ersten Schritt erfolgt die Segmentierung der Nutzergruppe nach zweckmäßigen Kriterien. Zwingend notwendig dafür ist eine tiefe Kenntnis der Nutzergruppe. In dem von uns betrachteten Fall erschienen folgende drei Kriterien sinnvoll: Hierarchieebene, Nutzungspräferenzen und funktions-

\section{Visuelles Design greift zu kurz - die langfristige Effektivität von Dashboards bestimmt sich jenseits vom Frontend.}

\section{Zusammenfassung}

- Eine WHU-Studie beim SoftwareHersteller SAP hat nach den Erfolgsfaktoren für die langfristige Akzep$\operatorname{tanz}$ und Nutzung von Dashboards gefragt.

- Die Ergebnisse der Studie unterstreichen die Dringlichkeit, sich von der Fokussierung auf visuelles Design zu lösen und stattdessen prozessuale Aspekte bei der Dashboard-Entwicklung und -Implementierung stärker zu berücksichtigen.

- Vier Empfehlungen können Unternehmen helfen, langfristig effektive, adaptive Dashboards bereitzustellen. 


\section{Ein statisches Verständnis von Dashboards als Produkt passt nicht zur Dynamik heutiger Unternehmenssteuerung.}

spezifische Aufgabenbündel. Die Hierarchieebene bestimmt in der Regel die benötigte Aggregationsstufe und den Zeithorizont der darzustellenden Informationen. Die Nutzungspräferenzen können Aufschluss darüber geben, ob es sich um Gelegenheits- oder sogenannte „Power-User“ handelt, oder wie erkundungsfreudig Nutzergruppen sind. Sie können über Verfahren wie Process Mining ermittelt werden. Auch über funktionsspezifische und weitverbreitete Aufgabenbündel (wie beispielsweise im HR-Bereich die Aufgabenbündel „Stelle ausschreiben“ und „Bewerber einladen“), die Dutzende oder Hunderte Male in der Organisation vorkommen, kann die Nutzergruppe segmentiert werden.

\section{„Eine mögliche Lösung für das Problem der Nutzerheterogenität kann eine nutzersegment- basierte Modularisierung des Dashboards sein."}

Im zweiten Schritt, nach Segmentierung entlang der zuvor festgelegten Kriterien, wird das Dashboard konzeptionell in Kern und Peripherie unterteilt: Der Kern umfasst alle Kennzahlen, die die Unternehmensstrategie operationalisieren und damit segmentunabhängig gelten. Die Peripherie variiert segmentabhängig in ihren Inhalten und kann beispielsweise für die jeweilige Bereichs- oder Abteilungsstrategie relevante Kennzahlen abbilden. Eine solche Unterscheidung zwischen Dashboard-Kern und -Peripherie fördert die wünschenswerte einfache und intuitive Nutzung und gleichzeitig die für kollektive Entscheidungsprozesse notwendige Standardisierung (vergleiche Yigitbasioglu/Velcu 2012, S. 51 ff.; Weber/Wiegmann 2018, S. 24 f.).

\section{Kontinuierliche Befähigung zur optimalen Nutzung}

Unsere Studie zeigte weiterhin, dass einige Nutzer die Struktur und Visualisierung des Dashboards hinterfragten und sich mit dessen Verwendung schwertaten. Einigen Nutzern war nicht hinreichend klar, welches Geschäftsproblem sie konkret mit dem Dashboard lösen könnten beziehungsweise weshalb bestimmte Kennzahlen für das Dashboard ausgewählt worden waren. Andere hatten Probleme, die Bedeutung des Dashboards im breiteren Unternehmenskontext zu verstehen. Ferner konnten sie keinen klaren Bezug vom Dashboard zu ihren individuellen Führungsaufgaben herstellen. Da es den Nutzern schwerfiel, implizites Erfahrungswissen zu verbalisieren, konnte es nicht in die Entwicklungsprozesse einfließen, sodass Potenziale in der Dashboard-Entwicklung ungenutzt blieben. Dies wiederum wirkte sich negativ auf die Akzeptanz aus.

\section{Empfehlung 3}

Dieser Befund macht deutlich, wie entscheidend gerade die subjektive Wahrnehmung für die tatsächliche Nutzung von Technologien ist (vergleiche dazu auch Davis 1989). Der Nutzer muss die Dashboard-Lösung von Anfang an 
als praktisch und nützlich für die Ausführung einer spezifischen Aufgabe wahrnehmen. Er muss schnell erfassen können, wie aufwendig es ist, die Nutzung eines Dashboards zu erlernen - und dieser Aufwand sollte möglichst gering sein. Mittlerweile können gerade bei der grafischen Gestaltung automatisiert einheitliche Standards wie beispielsweise die International Business Communication Standards (IBCS) genutzt werden und es so erleichtern, sich schnell in unterschiedlichen Dashboards zurechtzufinden. Dies trägt zu einer positiven Nutzererfahrung bei, die zur weiteren Nutzung motivieren kann (vergleiche Faisst 2021, S. 18 ff. in diesem Heft).

Die Verständlichkeit und Funktionalität der Lösung sowie ihre Relevanz für die eigene Arbeit beeinflussen die Einstellung der Nutzer gegenüber Dashboards. Stimmen aus deren Sicht alle Faktoren, erhöht sich die Wahrscheinlichkeit, dass sie ein neues Dashboard nutzen. Zusätzlich sind auch Experimentierfreude und eben doch auch Frontend-Ästhetik nicht zu unterschätzende Nutzungsmotivatoren, die in der Dashboard-Entwicklung explizit berücksichtigt werden sollten.

Im Entwicklungsprozess sollten immer die Bedürfnisse der intendierten Nutzer berücksichtigt und kontinuierlich reflektiert werden. Folgende Fragenkomplexe können dabei als Leitfaden dienen:

- Welches Ziel hat die Einführung des Dashboards? Was soll sich für die Nutzer ändern beziehungsweise verbessern?

- Kann das Dashboard die Arbeit der Nutzer vereinfachen? Welche Funktionalitäten sind dafür notwendig? Ist die Funktionsweise des Dashboards leicht verständlich und erlernbar? Wie lange dauert der Einarbeitungsprozess?

Diese Fragen müssen immer wieder gestellt werden - über die gesamte Lebensdauer des Dashboards gerade auch in Bezug auf hochkarätige Zielgruppen wie das obere Management. Hier helfen einerseits gutes ProjektManagement, andererseits auch regelmäßige Interviews und „Usability“-Tests (vergleiche Wouters/Wilderom 2008, S. 510 ff.). Datengetriebene Ansätze können zusätzliche Einblicke generieren, etwa mithilfe von Nutzungsstatistiken wie etwa denen, die SAP WebAnalytics bietet. Auch gezielte Umfragen unter den Nutzern im Rahmen der Anwendungsausführung können helfen. So können kontinuierlich Dashboard-Struktur und -Inhalte geprüft und verbessert werden.

\section{Keine Kompromisse bei der Technik}

In unserer Studie wurde auch deutlich, dass neben Entwicklungsprozessen und Design auch weithin bekannte, aber immer wieder stiefmütterlich behandelte Faktoren wie Verarbeitungsgeschwindigkeit, Datenaktualität und Datenverfügbarkeit die Nutzerzufriedenheit und damit die DashboardAkzeptanz beeinflussten. Wichtig waren den befragten Nutzern besonders die Reaktionszeit des Dashboards auf Anfragen und die Geschwindigkeit der Anwendung. „In einem Meeting sind zehn Sekunden eine Ewigkeit“, brachte es ein Nutzer auf den Punkt. Die Nutzer wollten über die Datenquelle informiert sein, um Vertrauen in die Qualität der Frontend-Zahlen haben zu können. Das in unserer Studie fokussierte Dashboard wurde beispiels-
Dashboarding appelliert und sensibilisiert dafür, Dashboards als kontinuierlich produziert zu begreifen. 


\section{Handlungsempfehlungen}

- Vermitteln Sie klar Zweck und Relevanz von Dashboards, um den Wertbeitrag für Nutzer herauszustellen, aber auch die Verbindlichkeit von Steuerungsinhalten aufzuzeigen.

- Unterscheiden Sie Dashboard-Kern und -Peripherie, um Nutzergruppen differenziert anzusprechen, ohne die für Steuerungseffekte wichtige Standardisierung zu gefährden.

- Befähigen Sie Mitarbeiter und Dashboard kontinuierlich zur optimalen Nutzung, um die Nutzerzufriedenheit zu maximieren und den langfristigen Fit des Dashboards mit veränderlichen Anforderungen und Steuerungskontexten $\mathrm{zu}$ sichern.

- Gehen Sie keine Kompromisse bei der Technik ein, damit mangelnde Performanz nicht alle anderen Bemühungen kompromittiert. weise sowohl aus den Datenbanken des Controllings als auch der Personalabteilung versorgt, in denen Kennzahlen verschieden definiert sein konnten. Generell wurde deutlich, dass eine komplexe Landschaft aus Informationssystemen eine besondere Sorgfalt bei der Herstellung und Kultivierung von Datenqualität erfordert.

\section{Empfehlung 4}

Seit der Verbreitung mobiler Endgeräte haben wir uns zunehmend daran gewöhnt, Technologie jenseits stationärer Computer stark in unseren Alltag zu integrieren. Unsere Erfahrungen im Alltag wirken sich auch auf unser Arbeitsumfeld aus und verändern Erwartungen an Geschäftsanwendungen. Im Reporting gelten Ästhetik, Schnelligkeit und nahtlose Übergänge zwischen verschiedenen Geräten zunehmend nicht mehr als Kann-, sondern als Muss-Kriterien. Erst die Erfüllung von Muss-Kriterien stellt die Voraussetzungen für die Verbreitung von Dashboards und für ihre Akzeptanz sowie Einbindung in den digitalen Arbeitsalltag her. Die Reaktionszeit auf Nutzeranfragen und die Schnelligkeit in der Interaktion entscheiden über den Grad der Nutzung einer Dashboard-Lösung. Um Frustrationen zu vermeiden, müssen Standards für Reaktionszeiten festgelegt und kontinuierlich überprüft werden. Ein langsames Dashboard sollte nicht für einen Roll-out freigegeben werden. Hier bedarf es ständiger kritischer Evaluation der Informationssysteme, die Dashboards mit Daten versorgen. Eine solche Evaluation darf nicht nur im Einzelnen erfolgen. Sie muss die gesamte Landschaft der unternehmensweit verwendeten Informationssysteme berücksichtigen, um eine Vereinheitlichung des Berichtswesens fördern zu können. Die Kosten von Informationssystemen sind für viele Ausgangspunkt von Investitionsdiskussionen geworden, in denen auch Geschwindigkeit in der Informationsverarbeitung zunehmend Beachtung findet. Es ist wichtig, Kennzahlen-Relevanz und Verarbeitungsgeschwindigkeit gebührend in Kommunikationskampagnen herauszustellen. Was bei Prozessdokumentationen heute Standard ist, muss auch für die interne Dashboard-Vermarktung gelten.

\section{Schlussbetrachtung}

Dashboards erfordern Überlegungen, die weit über visuelles Design hinausgehen und sich auf den gesamten Entwicklungs- und Implementierungsprozess erstrecken. Insbesondere die klare Vermittlung von Zweck und Relevanz, eine Unterscheidung zwischen Kern und Peripherie, die kontinuierliche Befähigung zur optimalen Nutzung sowie eine kompromisslose Priorisierung technischer Leistungsfähigkeit entscheiden über den Erfolg und die Nutzung von Dashboards. Allerdings glauben wir, dass diese Empfehlungen allein nicht ausreichen, um langfristig die Effektivität von Dashboards zu sichern. Vielmehr muss sich das Verständnis von Dashboards selbst weiterentwickeln. Dashboards werden noch zu häufig statisch als Produkt verstanden. Bleibt es dabei, besteht angesichts des stetigen Wandels technologischer Infrastrukturen, organisationaler Steuerungskontexte, des 
ständigen Wettbewerbs mit anderen Tools sowie veränderlicher Nutzerpopulationen die Gefahr, dass einmal implementierte Dashboards ihre Relevanz verlieren.

Wir fordern daher, über prozessbezogene Empfehlungen hinauszugehen und Dashboards selbst als Prozess zu verstehen - und damit eine Verschiebung der Diskussion von Dashboards zu „Dashboarding“. Mit diesem Begriff wollen wir die Prozesshaftigkeit von Dashboards unterstreichen, wonach Dashboards als kontinuierlich produziert zu verstehen sind. Erstens schafft Dashboarding als Begriff ein Bewusstsein dafür, dass Nutzer ständig neu überzeugt, Funktionen hinterfragt und die Verbindung zur Strategie immer wieder neu ausgelotet werden müssen. Zweitens vermeidet Dashboarding eine Überfokussierung auf das sichtbare, greifbare Frontend und die damit oft einhergehende Vernachlässigung wichtiger prozessualer Gestaltungsvariablen. Drittens schließlich kann Dashboarding auch eine kritische Analyse der Notwendigkeit zusätzlicher Tools begünstigen: Die „kontinuierliche Produktion“ von Dashboards kann, ernsthaft betrieben, mit einem nicht zu unterschätzenden Wartungs- und Entwicklungsaufwand einhergehen. Dies ermutigt dazu, lieber einige wenige, aber sorgfältig durchdachte und tatsächlich wirksame Dashboards zu entwickeln und langfristig zu pflegen, statt Unternehmen und Mitarbeiter mit „einfach bloß schönen Charts“ zu überfluten.

\section{„Wir fordern, Dashboards selbst als Prozess zu verstehen."}

Die von uns formulierten Empfehlungen sind nicht als Patentrezept für erfolgreiches Dashboarding zu verstehen. Vielmehr müssen Entscheider ihre Sinnhaftigkeit, Machbarkeit und konkrete Operationalisierung stets im spezifischen Unternehmenskontext betrachten. Einige wichtige Kontextelemente sind hier die Technologie, die Unternehmensstrategie und die Besonderheiten lokaler Nutzergruppen. Gerade mit Blick auf den anhaltend euphorischen Diskurs um Technologie gilt, dass Dashboarding von kritischer, informierter Reflexion begleitet sein sollte. Gutes Dashboarding ist Maßfertigung, nicht Fließbandarbeit. Eine gründliche Reflexion setzt auch ein Bewusstsein dafür voraus, dass Dashboards allein noch keine Strategie darstellen (vergleiche Weber/Schäffer 1998, S. 361). Vielmehr müssen sie immer wieder neu im strategischen Steuerungskontext der Organisation verankert werden. Eine solche Verankerung erfordert konzeptionelle Überlegungen, die eine Fixierung auf Dashboards als statisches Produkt überwinden müssen. Unser Aufruf zum Dashboarding und unsere Empfehlungen können dazu beitragen. 


\section{Literatur}

Davis, F. D. (1989): Perceived usefulness, perceived ease of use, and user acceptance of information technology, in: MIS Quarterly, 13 (3), S. 319-340.

Faisst, J. (2021): Dynamische Dashboards nach IBCS gestalten, in: Controlling \& Management Review, 65 (4), S. 18-25.

Gartner (2020): Market Share: analytics and business intelligence, worldwide, 2019, https://tinyurl.com/analytics-and-BI-worldwide (letzter Abruf: 09.03.2021).

Johns Hopkins University (2020): COVID-19 dashboard. Center of Systems Science and Engineering (CSSE), https://coronavirus.jhu.edu/map.html (letzter Abruf: 09.03.2021).

Reimer, M./Schäffer, U./Weber, J. (2019): Erfolgreiches Management Reporting. Eine Studie des WHU Controller Panels, Vallendar.

Sabherwal, R./Jeyaraj, A./Chowa, C. (2006): Information system success: individual and organizational determinants, in: Management Science, 52 (12), S. 1849-1864.

Schäffer, U./Weber, J. (2016): Die Digitalisierung wird das Controlling radikal verändern, in: Controlling \& Management Review, 60 (6), S. 6-17.

www.springerprofessional.de/link/11914878

Simons, R. (1995): Levers of control. How managers use innovative control systems to drive strategic renewal, Boston.

Tufte, E. R. (2001): The visual display of quantitative information, 2. Auflage, Cheshire.

Weber, J./Schäffer, U. (2020): Einführung in das Controlling, 16. Auflage, Stuttgart.

Weber, J./Schäffer, U. (1998): Balanced Scorecard. Gedanken zur Einordnung des Konzepts in das bisherige Controlling-Instrumentarium, in: Zeitschrift für Planung \& Unternehmenssteuerung, 9 (4), S. 341-365.

Weber, J./Wiegmann, L. (2018): Self-Service BI. Mehr Personalisierung oder bewährte Standardisierung des MIS?, in: Wirtschaftsinformatik \& Management, 10 (3), S. 20-25.

www.springerprofessional.de/link/15844388

Wouters, M./Wilderom, C. (2008): Developing performance-measurement systems as enabling formalization: a longitudinal field study of a logistics department, in: Accounting, Organizations and Society, 33 (4-5), S. 488-516.

Yigitbasioglu, O. M./Velcu, O. (2012): A review of dashboards in performance management: implications for design and research, in: International Journal of Accounting Information Systems, 13 (1), S. 41-59.

\section{照 Springer Professional}

Proff, H. et al. (2021): Accelerating Digitalization - Chancen der Digitalisierung erkennen und nutzen, Wiesbaden. www.springerprofessional.de/link/18985700

Schlüter, A. (2015): Mit Dashboards navigieren, in: Controlling \& Management Review, 59 (Sonderheft 3), S. 30-35.

www.springerprofessional.de/link/7031192 


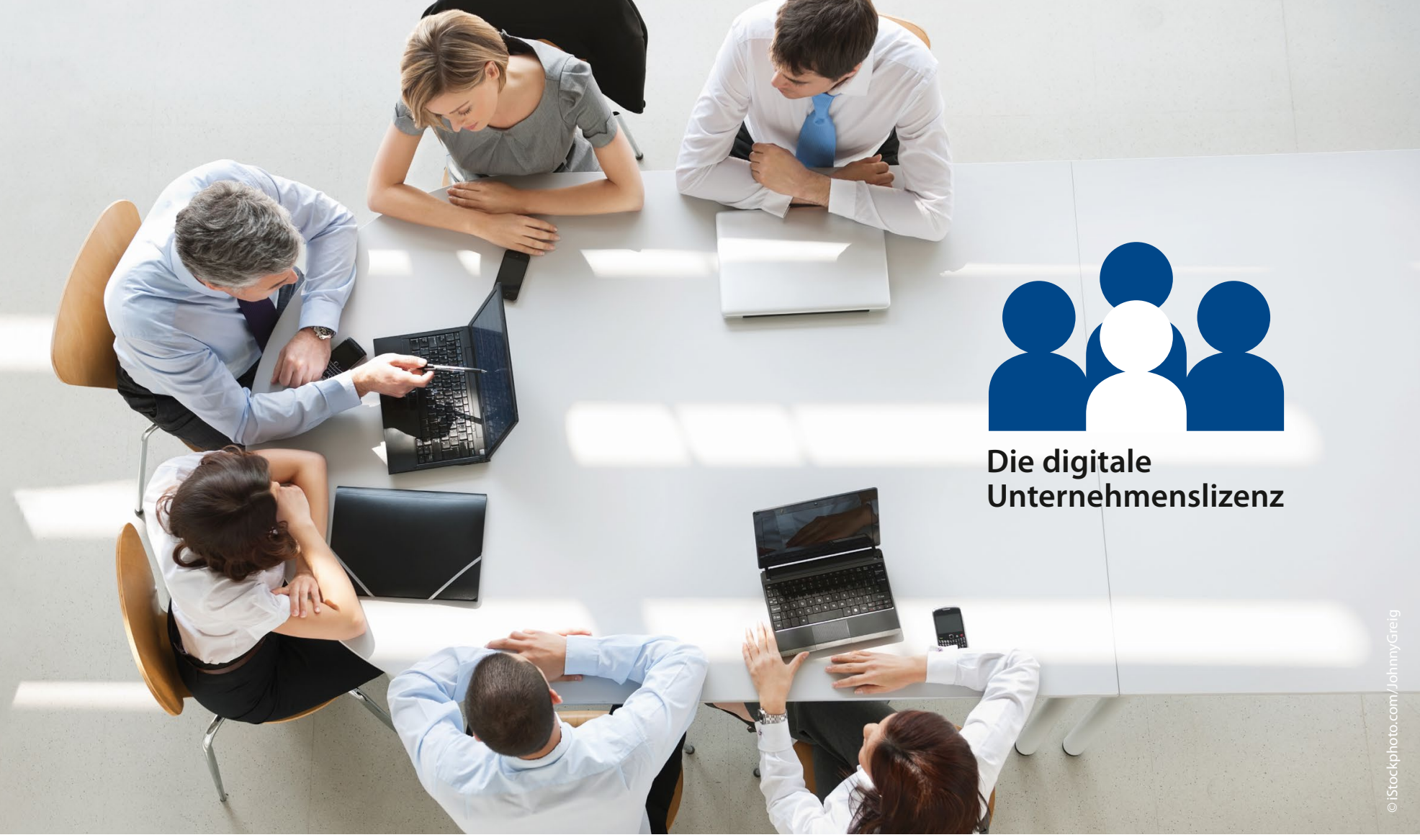

\section{Fundiertes Fachwissen für Ihr Team!}

Profitieren Sie und Ihre Mitarbeiter von dem geballten Fachwissen von Controlling \& Management Review. Mit einer digitalen Unternehmenslizenz erhalten Sie vollen Zugriff auf die interaktiven E-Magazine und das umfangreiche Online-Archiv. Wählen Sie Ihr individuelles Zugangsmodell für Ihre Team- bzw. Unternehmensgröße - von IP-Freischaltung bis individuellem Login.

$\checkmark$ Umfassend: aktuelle Ausgaben plus Fachartikelarchiv seit 2000 $\checkmark$ Kostensparend: exklusive Rabatte für Ihr Team

$\checkmark$ Individuell: das passende Zugangsmodell für Ihre Bedürfnisse $\checkmark$ Flexibel: jederzeit und überall lesen

$\checkmark$ Interaktiv: zusätzliches Spezialwissen durch verlinkte Inhalte $\checkmark$ Rechtssicher: erfüllt das Urheber- und Lizenzrecht

Wir beraten Sie gern: Ramona Wendler

Tel. 0611 7878-126 | magazinlizenzen@springernature.com

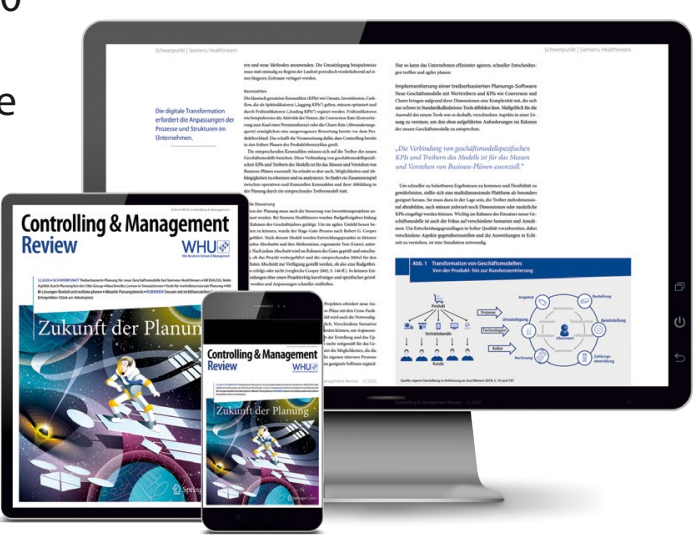

Controlling \& Management Review 